\title{
Identification of Novel Target Proteins of Cyclic GMP Signaling Pathways Using Chemical Proteomics
}

\author{
Euikyung Kim* and Ji Man Park \\ Department of Life Science, Division of Molecular and Life Science, Pohang University of Science \& Technology, \\ Pohang, Kyungbuk 790-784, Republic of Korea
}

Received 18 November 2002, Accepted 10 January 2003

For deciphering the cyclic guanosine monophosphate (cGMP) signaling pathway, we employed chemical proteomics to identify the novel target molecules of cGMP. We used cGMP that was immobilized onto agarose beads with linkers directed at three different positions of cGMP. We performed a pull-down assay using the beads as baits on tissue lysates and identified 9 proteins by MALDI-TOF (Matrix-Assisted Laser Desorption/Ionization Time-ofFlight) mass spectrometry. Some of the identified proteins were previously known cGMP targets, including cGMPdependent protein kinase and cGMP-stimulated phosphodiesterase. Surprisingly, some of the coprecipitated proteins were never formerly reported to associate with the cGMP signaling pathway. The competition binding assays showed that the interactions are not by nonspecific binding to either the linker or bead itself, but by specific binding to cGMP. Furthermore, we observed that the interactions are highly specific to cGMP against other nucleotides, such as cyclic adenosine monophosphate (cAMP) and 5'-GMP, which are structurally similar to cGMP. As one of the identified targets, MAPK1 was confirmed by immunoblotting with an anti-MAPK1 antibody. For further proof, we observed that the membrane-permeable cGMP (8-bromo cyclic GMP) stimulated mitogen-activated protein kinase 1 signaling in the treated cells. Our present study suggests that chemical proteomics can be a very useful and powerful technique for identifying the target proteins of small bioactive molecules.

Keywords: Agarose bead, Chemical immobilization, Competition binding, Cyclic GMP, MALDI mass spectrometry

*To whom correspondence should be addressed. Tel: 82-54-279-2973; Fax: 82-54-279-2199

E-mail: ekim@postech.ac.kr

\section{Introduction}

Upon the completion of the Human Genome Project, we now have access to the full human DNA sequence information. However, it is difficult to understand the functional significance and regulation of the genes that interest us by their DNA sequence alone (Clark, 1999). Chemical proteomics (often called chemical genetics or chemical genomics) are a powerful methodology for deciphering the gene functions (Hung et al., 1996; You et al., 1997; Shirai et al., 1998; Bergseid et al., 2000; Chan et al., 2000).

Cyclic nucleotides, e.g. cyclic AMP (cAMP) and cyclic GMP (cGMP), are universal second messengers like $\mathrm{Ca}^{2+}$ and have involved in a multitude of cellular events such as transcription regulation, chemotaxis, proliferation, differentiation, and apoptosis. Presently, there are myriad analogues that have been synthesized with different biological activities for deciphering cyclic nucleotide signaling pathways. However, only a few have been explored with regard to their therapeutic potential such as anticancer agents or therapeutics against diseases of the immune system (for review see Schwede et al., 2000). Cyclic GMP is synthesized by guanylyl cyclases in response to diverse signals such as nitric oxide (NO), peptide ligands, and fluxes in intracellular $\mathrm{Ca}^{2+}$. The specificity of cellular responses to cGMP is dictated by cGMP-binding motifs in target proteins. Two evolutionarily distinct allosteric sites for binding cGMP are present in eukaryotic cells. One occurs with significant sequence homology in cyclic GMP-dependent protein kinases (PKGs), cAMP-dependent protein kinases A (PKAs), and cyclic nucleotide-gated cation channels; the other occurs in cGMP-regulated phosphodiesterases (PDEs). Intracellular cGMP regulates cellular physiology by activating protein kinases, directly gating specific ion channels, or altering intracellular cyclic nucleotide concentrations through the regulation of PDEs.

The multitude of well-known target proteins for cyclic nucleotides, such as PDEs, cNMP-regulated protein kinases, 
and ion channels, in combination with the potential crosstalk at several steps of the cAMP and cGMP signaling cascades represents the enormous target complexity. None of the common cyclic nucleotide analogs have as yet been tested with every isozyme that is involved in cAMP and cGMP signaling. Despite this target complexity, several cNMP analogs have shown excellent properties as tools in signal transduction research in vitro and in vivo, and even in some important clinical applications. Although cGMP has been extensively studied as a downstream signaling molecule of nitric oxide (Choi et al., 2002; Kim et al., 2002), their molecular targets have not been completely characterized, particularly the ones that are regulated by the direct binding of cGMP. In the present study, we applied the chemical proteomics technique to identify the cGMP binding proteins for deciphering cGMP signaling pathways.

\section{Materials and Methods}

Materials The cGMP coupled agarose beads were purchased from the BIOLOG Life Science Institute (Bremen, Germany). BioRad Silver Stain Kit was from Bio-Rad (Hercules, USA). Protease inhibitor mixture tablets were purchased from Roche Diagnostics (Basel, Switzerland). The rabbit polyclonal anti-phospho MAPK antibody was from Cell Signaling Technology, Inc. (Beverly, USA). All of the other chemicals were purchased from Sigma (St. Louis, USA).

Tissue lysates preparation After sacrificing the rats (Sprague Dawley, $200 \mathrm{~g}$ ), their brains, hearts (perfused), and livers were removed and processed for the preparation of tissue lysates. Briefly, the brain was homogenized using a polytron glass homogenizer $(20 \mathrm{~s}$, twice) in a $5 \times(\mathrm{v} / \mathrm{w})$ homogenization buffer $(50 \mathrm{mM}$ Tris$\mathrm{HCl}, \mathrm{pH} 7.5,50 \mathrm{mM} \mathrm{NaCl}, 1 \mathrm{mM}$ EDTA/EGTA, $50 \mathrm{mM} \mathrm{NaF}$, $5 \mathrm{mM}$ sodium pyrophosphate, $1 \mathrm{mM}$ PMSF, protease inhibitor mixture, $1 \mathrm{mM}$ DTT). The liver and heart were minced and homogenized using a polytron homogenizer instead of a glass homogenizer under the same conditions. The tissue homogenates were centrifuged $(100,000 \times g, 1 \mathrm{~h})$, then the supernatant were collected. The protein concentrations of the tissue lysates were determined by a Bradford assay and used for the pull-down assay.

Pull-down assay Cyclic GMP-coupled agarose beads $(50 \mathrm{nmol}$ of cGMP) were washed with a washing buffer $(50 \mathrm{mM}$ Tris- $\mathrm{HCl}$, pH 7.5, $50 \mathrm{mM} \mathrm{NaCl}, 1 \mathrm{mM}$ EDTA/EGTA, $50 \mathrm{mM} \mathrm{NaF}, 5 \mathrm{mM}$ sodium pyrophosphate, $1 \%$ Triton $\mathrm{X}-100$ ) before use. For the control beads, CL-4B agarose beads were used and prepared in the same way. The cGMP-coupled beads, as well as the control beads, were incubated with tissue lysate $\left(5 \mathrm{mg} /\right.$ reaction) at $4^{\circ} \mathrm{C}$ for $2 \mathrm{~h}$. Non-specifically bound proteins to the beads were removed by rinsing with a washing buffer three times, then a SDS sample buffer was added to the beads and boiled for $5 \mathrm{~min}$ at $95^{\circ} \mathrm{C}$. The proteins that bound to the beads with high affinity were separated by SDSPAGE (6-16\%) and visualized by silver staining.

Competition binding assay For validating the specificity of the interaction between the proteins and chemical, we performed a competition binding assay, which is generally employed in a receptor ligand binding study. Briefly, the indicated concentrations of free ligand (cGMP, cAMP, or GMP) were added to the reaction mixture of the pull-down assay. The principle of this competition binding assay is that the presence of excess amounts of free (or unlabeled) ligand inhibits the ligand specific interaction of the receptor with its bead-coupled (or labeled) ligand by competition.

Protein identification by peptide mass fingerprinting analysis Fractions of the phenyl-Sepharose were subjected to SDS-PAGE. After silver staining, the candidate band was excised from the gel and digested with trypsin, as described (Jensen et al., 1996). A 1- $\mu 1$ aliquot of the total digest (total volume, $30 \mu \mathrm{l}$ ) was used for the peptide mass fingerprinting (Mortz et al., 1994; Shevchenko et al., 1996). The tryptic peptides masses were measured with a Bruker REFLEX III time-of-flight mass spectrometer at the Pohang University of Science and Technology. Matrix-assisted laser desorption/ionization was performed with $\alpha$-cyano-4hydroxycinnamic acid as the matrix. Trypsin autolysis products were used for the internal calibration. Delayed ion extraction resulted in peptide masses with better than $50 \mathrm{ppm}$ mass accuracy on average. Comparison of the mass values against the NCBInr database was performed using Peptide Search.

Immunoblotting The initial sample for immunoblotting was prepared in the same way as for silver staining. After a run on the 6$16 \%$ SDS-PAGE, the separated proteins were transferred to nitrocellulose membranes (Schleicher and Schuell, Keene, USA) instead of silver staining. Blocking was performed with a TTBS buffer (10 mM Tris-HCl, pH 7.6, $150 \mathrm{mM} \mathrm{NaCl}, 0.1 \%$ Tween 20$)$ containing 5\% skimmed milk. The membranes were then incubated with a mouse monoclonal anti-MAPK antibody for $4 \mathrm{~h}$ at room temperature. The immunoblots were washed for $2 \mathrm{~h}$, changing the TTBS buffer every $20 \mathrm{~min}$, then incubated with a horseradish peroxidase-linked secondary antibody for $30 \mathrm{~min}$ at room temperature. The immunoblots were washed every $10 \mathrm{~min}$ for $1 \mathrm{~h}$ with a changing TTBS buffer, and developed with horseradish peroxidase-dependent chemiluminescence (Amersham Corp., Piscataway, USA).

Cyclic GMP-dependent MAPK1 activation After serum starvation $(24 \mathrm{~h})$, the HeLa S cells were treated for $10 \mathrm{~min}$ with membrane permeable 8 -Br-cGMP in the presence or absence of EGF. The activation of MAPK1 was determined by immunoblotting with an anti-phospho MAPK1 antibody. Briefly, following the cGMP treatment, the cells were washed with PBS and added with a lysis buffer (1\% Triton X-100, $150 \mathrm{mM} \mathrm{NaCl}$, $20 \mathrm{mM}$ Tris- $\mathrm{HCl}, \quad \mathrm{pH} \quad 7.4,50 \mathrm{mM} \mathrm{NaF}, 1 \mathrm{mM}$ sodium orthovanadate, $1 \mathrm{mM}$ PMSF, $1 \mu \mathrm{g} / \mathrm{ml}$ leupeptin, $5 \mu \mathrm{g} / \mathrm{ml}$ aprotinin, and $2 \mu \mathrm{M}$ pepstatin A) on ice. The crude cell lysates were scraped with a rubber policeman, then transferred to eppendorf tubes and kept on ice for $25 \mathrm{~min}$ with intermittent vigorous vortexing. The supernatants were obtained after centrifugation at 15,000 rpm for $10 \mathrm{~min}$ at $4^{\circ} \mathrm{C}$, and their protein concentrations were determined using a Bradford assay. The cell lysates were added with $(5 \times)$ a SDS sample buffer and heated at $95^{\circ} \mathrm{C}$ for $5 \mathrm{~min}$. The proteins were separated by $6-16 \%$ SDS-PAGE, and transferred to nitrocellulose 
<smiles>Nc1nc2c(nc(SCCNO)n2C2OCC3O[P+](=O)([O-])OC3O2)c(=O)[nH]1</smiles>

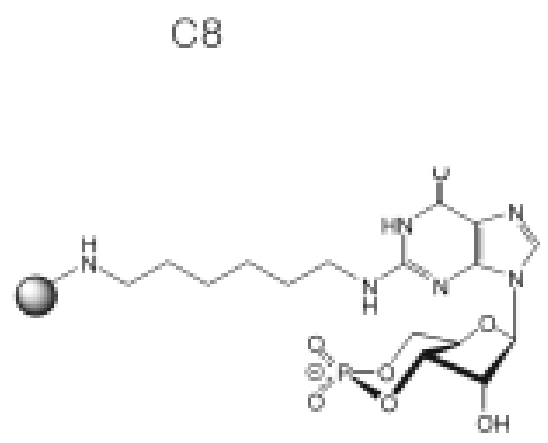

N2

Fig. 1. The cGMP-immobilized agarose beads. Cyclic GMP molecules were coupled to agarose beads using linkers which were directed at various positions of cGMP. Depending on the coupling positions in the structure of cGMP, they were named $\mathrm{C} 8, \mathrm{~N} 2$, and $\mathrm{O} 2$.

membranes (Schleicher and Schuell, Keene, USA). Immunoblotting was performed with a rabbit polyclonal antiphospho MAPK antibody as described above.

\section{Results and Discussion}

For the last several years, we have built up a MALDI-TOF mass spectrometry, a cutting edge tool for peptide identification from biological samples. We recently expanded the mass spectrometry operation by establishing chemicalproteomics methodology, which is a powerful technique for discovering the target proteins of small bioactive chemicals. Compared with cAMP signaling, the cGMP signaling pathways have not been understood well. In order to decipher the cGMP signaling mechanisms, we sought to discover the cGMP binding proteins.

Coupling Strategy of cGMP onto agarose beads We employed three different kinds of cGMP-coupled agarose beads, which are cyclic GMP molecules that have been chemically coupled to agarose beads using linkers at various positions of cGMP (Fig. 1). Depending on the coupling positions in the cGMP structure, we named them $\mathrm{C} 8, \mathrm{~N} 2$, and O2. Briefly, 8-AET-cGMP-Agarose (named C8) is the cGMP that is immobilized on agarose by an aminoethylthio spacer that is attached to position 8 of cGMP (Schlossmann et al., 2000). The 2-AH-cGMP-Agarose (named N2) is the cGMP

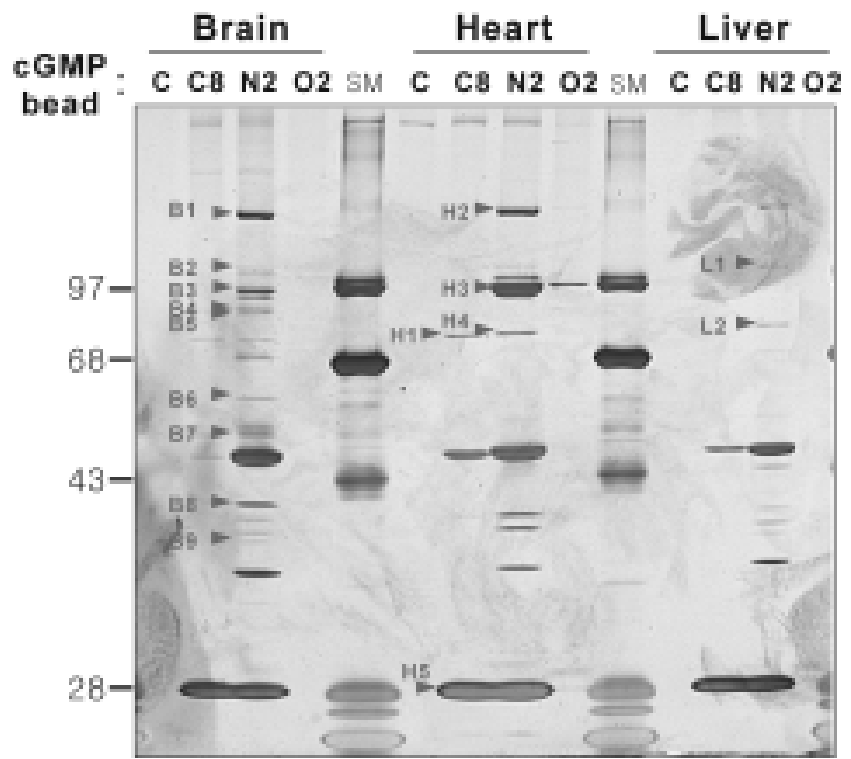

Fig. 2. Tissue specific protein profiles precipitated by cGMPcoupled beads. Following the incubation of cyclic GMP $(50 \mu \mathrm{M}$ final)-coupled beads with each of the tissue extracts $(5 \mathrm{mg}$ of protein $/ \mathrm{mL}$ ), the beads were pelleted by quick spin and washed 3 times with a fresh washing buffer, then added with the SDSPAGE sample buffer and heated at $95^{\circ} \mathrm{C}$ for $5 \mathrm{~min}$. The preparation and identification of the proteins were performed as described in Materials and Methods. Briefly, the pull-down proteins were separated by SDS-PAGE (6-16\% gradient), then visualized by silver staining. The protein bands were cut and analyzed by MALDI-TOF Mass Spectrometry (Matrix-Assisted Laser Desorption/Ionization Time-of-Flight Mass Spectrometry). Table 1 summarizes the proteins that were identified in. $\mathrm{C}=$ control bead; $\mathrm{C} 8, \mathrm{~N} 2$, and $\mathrm{O} 2=$ cGMP beads; $\mathrm{SM}=$ molecular weight size marker.

that is immobilized on agarose by an aminohexyl spacer that is attached to the 2-amino group of cGMP (Dills et al., 1976). The 2-AHC-cGMP-Agarose is the cGMP that is immobilized on agarose by an aminohexylcarbamoyl spacer that is attached to the ribose 2 position of cGMP. Hence, we expected the diversity of these coupling positions between cGMP and agarose beads to allow for the full accessibility of cGMP interaction with the various binding partners.

Isolation of cyclic GMP binding proteins The identification of the target protein(s) of a small molecule (either drug or toxicant) is not a simple task; it has been a laborious process in the traditional scientific approach. Upon the fusion of two very powerful technologies (chemical immobilization and MALDI-TOF mass spectrometry), we were able to accelerate the target identification of a small chemical of interest. Using these cGMP beads, we performed a pull-down assay against rat tissue lysates. From the experiment, we found that these beads interact with several proteins which seem to be very specific to cGMP (Fig. 2). A total of 9 out of 16 target proteins of cGMP were successfully 
Table 1. Proteins that were precipitated with cGMP-agarose beads in the brain, heart, and liver. (The result of MALDI-TOF mass spectrometry of Fig. 2 samples)

\begin{tabular}{llcccc}
\hline Label & \multicolumn{1}{c}{ Protein } & $\begin{array}{c}\text { Accession } \\
\text { Number }\end{array}$ & $\begin{array}{c}\text { MW } \\
(\mathrm{kDa})\end{array}$ & $\begin{array}{c}\text { Number of } \\
\text { Matches }\end{array}$ & $\begin{array}{c}\text { Sequence } \\
\text { Coverage }(\%)\end{array}$ \\
\hline B1 & Valyl-tRNA synthetase & AAD26531 & 140.28 & 31 & 12 \\
B2 & Cyclic-GMP stimulated phosphodiesterase & NP_112341 & 104.65 & 22 & 13 \\
B5 & keratin 1 & AAG41947 & 66.05 & 17 & 10 \\
B6 & calmodulin-dependent phosphodiesterase 1B & AAC96022 & 57.01 & 30 & 25 \\
B8 & Mitogen-activated protein kinase 1 & XP_009860 & 40.4 & 37 & 34 \\
H1 & cGMP-dependent protein kinase, type1 & NP_035290 & 77.77 & 29 & 18 \\
H2 & Valyl-tRNA synthetase & AAD26531 & 140.28 & 51 & 17 \\
H4 & cGMP-dependent protein kinase, alpha & Q13976 & 76.35 & 23 & 23 \\
L1 & Cyclic-GMP stimulated phosphodiesterase & NP_112341 & 104.65 & 16 & 9 \\
\hline
\end{tabular}

Proteins were isolated and analyzed as described in the text. Any match with a score (Est'd Z) greater than 1.5 was considered significant. NCBI accession numbers are for mammalian sequences

identified by MALDI-TOF mass spectrometry (Table 1). Among the identified, some (5 out of 9 identifications) are already well-known cGMP target proteins including cGMP stimulated phosphodiesterase (Essayan, 2001; Rotella, 2002) and cGMP-dependent protein kinase (Francis and Corbin, 1999; Hofmann et al., 2000). This implies that the proteins that are precipitated by the agarose-coupled cGMP of our present study are quite likely the target proteins of cGMP, as well as being regulated by cGMP. One of the interesting points in this result is that the N2 cGMP bead was capable of precipitating more proteins than other tested beads whereas the $\mathrm{O} 2$ cGMP bead was a poor bait for pulling down the binding partners. It implies that the interactions are highly stereo-specific, and that most of the binding pockets seem to lock in the cGMP molecule from the direction far from the N2 position of cGMP with a less steric hindrance. Another noteworthy point is that brain tissue has more cGMP binding proteins than the heart or liver, implying that cGMP has a more diverse signal transduction role in the brain than in the heart or liver.

Specificity of the interactions between cyclic GMP and target proteins For validating the binding specificity of the cGMP-target protein interactions, we performed a competition binding assay as described in Materials and Methods. Interestingly, we found that most of the cGMP binding proteins were efficiently dissociated by the addition of excess amounts of free cGMP to the pull-down assay (Fig. 3). On the other hand, the addition of excess amounts of free cAMP had a negligible effect on most of the interactions. This result implies that cGMP binds specifically to the cGMP specific binding sites that are present in the target proteins, and these are not shared by the other cyclic nucleotides for binding. Furthermore, most of the cGMP-target protein interactions were not deterred by 5-GMP, except a few such as ValyltRNA synthetase. This suggests that the binding site is highly specific to cGMP, even against 5-GMP. When we compare the

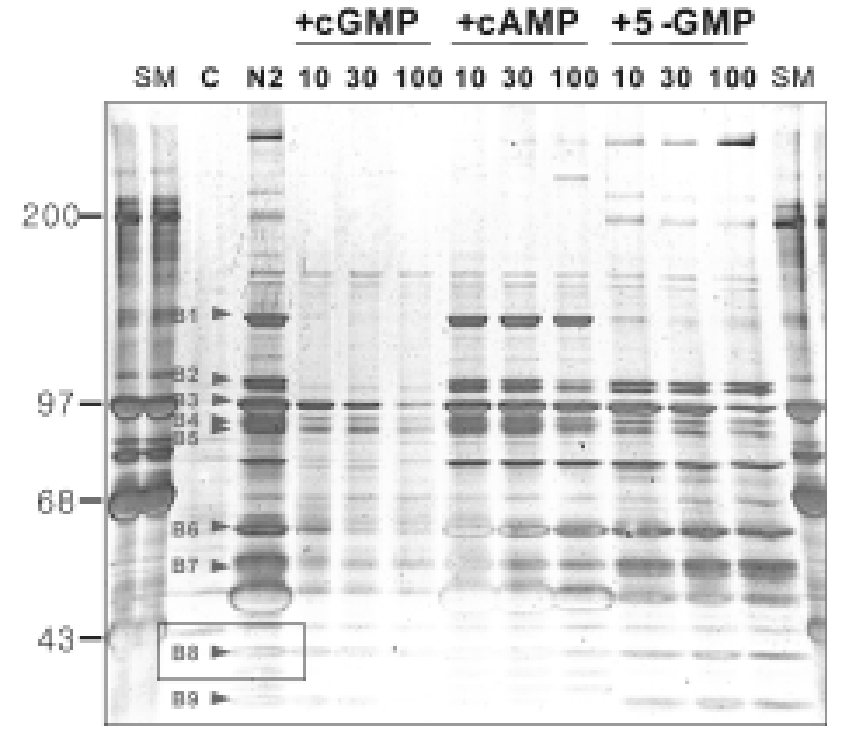

Fig. 3. Specificity of cGMP and protein interactions. Competition binding assays were performed as described in Materials and Methods. Briefly, the cGMP-coupled agarose beads (N2) were incubated with brain tissue lysate in the presence or absence of excess amounts of the free (uncoupled) nucleotides including cGMP, cAMP, and 5'-GMP. The numbers of fold excess of the free nucleotide when compared to the immobilized cGMP are indicated at the top of the figure. The preparation and identification of the proteins were performed as described in Materials and Methods. A box (B8) indicates the mitogen-activated protein kinase 1 (MAPK1) identified as a specific binding protein of cGMP. $\mathrm{SM}=$ molecular weight size marker; $\mathrm{C}=$ control bead; $\mathrm{N} 2=$ cGMP bead.

molecular structure of cGMP with those of cAMP and 5GMP, the similarity and dissimilarity between the structures is easily seen (Fig. 4). The structural differences between cAMP and cGMP are the amino residues at $\mathrm{C} 2$ and $\mathrm{C} 6$ of the purine ring (dotted area), which results in their protein binding profile 


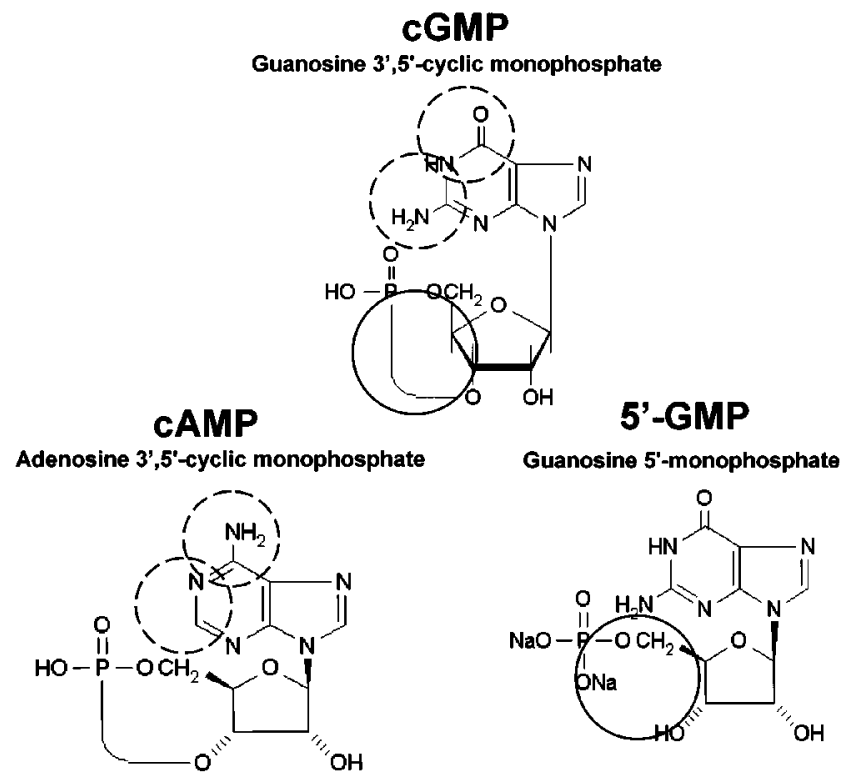

Fig. 4. Comparison of molecular structures of nucleotides. The dotted circles indicate the difference between cGMP and cAMP. A solid circle indicates the difference between cGMP and 5'GMP.

being totally different, as can be observed in this study. In addition, the structural difference between cGMP and 5-GMP is the cycle formation between the 3 and 5 position of the ribose ring (solid circle) of the nucleotides, which also shows distinctive preferences in their protein bindings. These results suggest that the cGMP molecule is locked into the binding pocket of its target proteins in a highly stereo-specific manner.

Activation of the mitogen-activated protein kinase 1 by cGMP For validating the MALDI-TOF mass spectrometry data, we immunoblotted the cGMP bead (N2) pull-down samples using a mouse monoclonal antibody against MAPK1, one of the identified targets (Fig. 5A). From the experiment, we observed the presence of MAPK1 in the precipitates of the cGMP beads, confirming the mass spectrometry result. In a further work for characterizing the MAPK1 binding, we tested whether the mitogen-activated protein kinase 1 (MAPK1) can be activated by cGMP (Fig. 5B). The treatment of the membrane permeable 8-bromo cyclic GMP showed a significant increase of MAPK phosphorylation that was detected by immunoblotting with an anti-phospho MAPK antibody.

In conclusion, using the chemical proteomics approach, we successfully identified several cGMP-binding proteins that have specific interactions with cGMP. Although the interactions can be either direct or indirect for cGMP, we currently suspect that they are direct. These binding proteins are possible targets of cGMP and are probably regulated by cGMP in the cells. We believe that our present work introduces chemical proteomics as a powerful method for deciphering small chemical-induced signaling pathways.

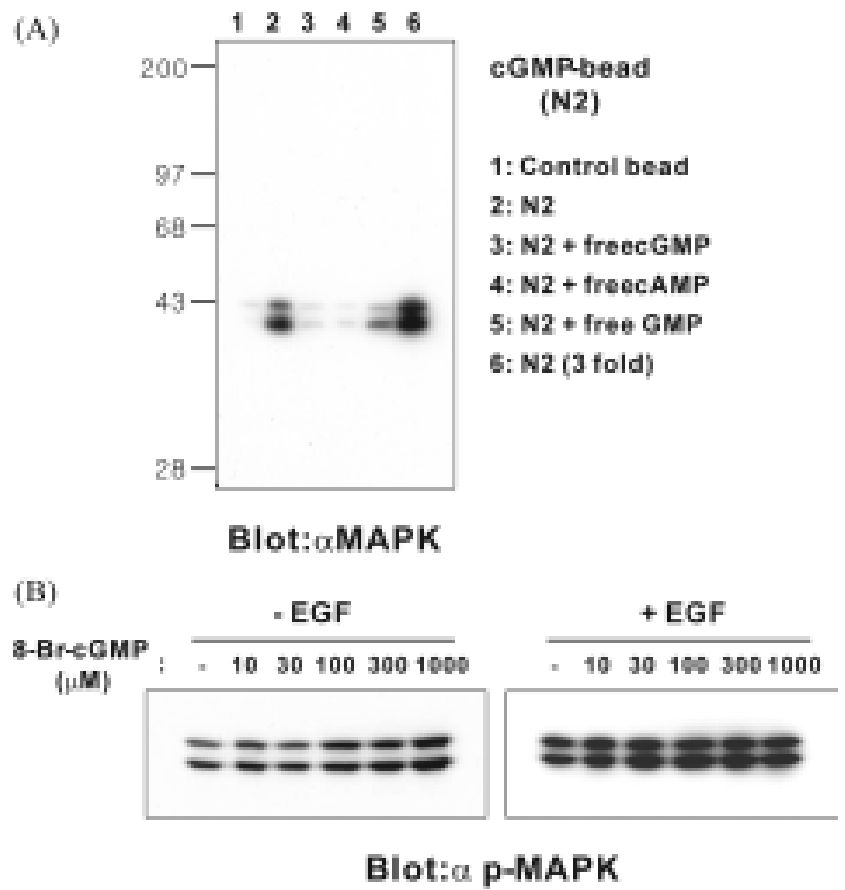

Fig. 5. Confirmation of the MALDI-TOF mass spectrometry result by MAPK1 immunoblotting and MAPK1 activation. (A) The identification of MAPK1 by MALDI-TOF mass spectrometry was confirmed by competition binding and immunoblotting. Briefly, the cGMP-agarose bead (N2) was incubated with brain tissue lysate in the presence or absence of 100 fold excess of free (uncoupled) nucleotides (described in Fig. 3). The samples were run on SDS-PAGE (6-16\% gradient), then immunoblotted with an anti-MAPK1 antibody. (B) The effect of cGMP on MAPK1 as its target protein was determined in HeLa S cells. Briefly, after serum starvation $(24 \mathrm{~h})$, the cells were treated for $10 \mathrm{~min}$ with membrane permeable 8 -Br-cGMP in the presence or absence of EGF. The activation of MAPK1 was determined by immunoblotting with an anti-phospho MAPK1 antibody.

Acknowledgments We thank the members of our laboratory, particularly Ho Young Lee for the excellent technical assistance as well as Drs. Pann-Ghill Suh and Sung Ho Ryu for the helpful discussions. We also thank Mr. Woo Young Hur for the initial contribution. This work was supported in part by a grant from the Korea Health 21 R\&D Project, Ministry of Health \& Welfare, Republic of Korea. (02-PJ2-PG4-PT01-0020).

\section{References}

Bergseid, M., Baytan, A. R., Wiley, J. P., Ankener, W. M., Stolowitz, M. L., Hughes, K. A. and Chesnut, J. D. (2000) Small molecule-based chemical affinity system for the purification of proteins. Biotechniques 29, 1126-1133.

Chan, T. F., Carvalho, J., Riles, L. and Zheng, X. F. (2000) A chemical genomics approach toward understanding the global 
functions of the target of rapamycin protein (TOR). Proc. Natl. Acad. Sci. USA 97, 13227-13232.

Choi, B. -M., Pae, H. -O., Jang, S. I., Kim, Y. -M. and Chung, H. -T. (2002) Nitric oxide as a pro-apoptotic as well as antiapoptotic modulator. J. Biochem. Mol. Biol. 35, 116-126.

Clark, M. S. (1999) Comparative genomics: the key to understanding the Human Genome Project. Bioessays 21, 121130.

Dills, W. L., Beavo, J. A., Bechtel, P. J., Myers, K. R., Sakai, L. J. and Krebs, E. G. (1976) Binding of adenosine 3',5'monophosphate dependent protein kinase regulatory subunit to immobilized cyclic nucleotide derivatives. Biochemistry 15, 3724-3730.

Essayan, D. M. (2001) Cyclic nucleotide phosphodiesterases. J. Allergy Clin. Immunol. 108, 671-680.

Francis, S. H. and Corbin, J. D. (1999) Cyclic nucleotidedependent protein kinases: intracellular receptors for cAMP and cGMP action. Crit. Rev. Clin. Lab. Sci. 36, 275-328.

Hofmann, F., Ammendola, A. and Schlossmann, J. (2000) Rising behind NO: cGMP-dependent protein kinases. J. Cell Sci. 113, 1671-1676.

Hung, D. T., Jamison, T. F. and Schreiber, S. L. (1996) Understanding and controlling the cell cycle with natural products. Chem. Biol. 3, 623-639.

Jensen, O. N., Vorm, O. and Mann, M. (1996) Sequence patterns produced by incomplete enzymatic digestion or one-step Edman degradation of peptide mixtures as probes for protein database searches. Electrophoresis 17, 938-944.

Kim, K. -M., Kim, P. K. M., Kwon, Y. -G., Bai, S. -K., Nam, W.
-D. and Kim, Y. -M. (2002) Regulation of apoptosis by nitrosative stress. J. Biochem. Mol. Biol. 35, 127-133.

Mortz, E., Vorm, O., Mann, M. and Roepstorff, P. (1994) Identification of proteins in polyacrylamide gels by mass spectrometric peptide mapping combined with database search. Biol. Mass Spectrom. 23, 249-261.

Rotella, D. P. (2002) Phosphodiesterase 5 inhibitors: current status and potential applications. Nat. Rev. Drug Discov. 1, 674-682.

Schlossmann, J., Ammendola, A., Ashman, K., Zong, X., Huber, A., Neubauer, G., Wang, G. X., Allescher, H. D., Korth, M., Wilm, M., Hofmann, F. and Ruth, P. (2000) Regulation of intracellular calcium by a signaling complex of IRAG, IP3 receptor and cGMP kinase Ibeta. Nature 404, 197-201.

Schwede, F., Maronde, E., Genieser, H. and Jastorff, B. (2000) Cyclic nucleotide analogs as biochemical tools and prospective drugs. Pharmacol. Ther. 87, 199-226.

Shevchenko, A., Wilm, M., Vorm, O. and Mann, M. (1996) Mass spectrometric sequencing of proteins silver-stained polyacrylamide gels. Anal. Chem. 68, 850-858.

Shirai, T., Tanaka, K., Terada, Y., Sawada, T., Shirai, R., Hashimoto, Y., Nagata, S., Iwamatsu, A., Okawa, K., Li, S., Hattori, S., Mano, H. and Fukui, Y. (1998) Specific detection of phosphatidylinositol 3,4,5-trisphosphate binding proteins by the PIP3 analogue beads: an application for rapid purification of the PIP3 binding proteins. Biochim. Biophys. Acta 1402, 292-302.

You, A. J., Jackman, R. J., Whitesides, G. M. and Schreiber, S. L. (1997) A miniaturized arrayed assay format for detecting small molecule-protein interactions in cells. Chem. Biol. 4, 969-975. 